\title{
Localized Asymmetric Atomic Matter Waves in Two-Component Bose-Einstein Condensates Coupled with Two Photon Microwave Field
}

\author{
Bo Xiong \\ Beijing National Laboratory for Condensed Matter Physics, \\ Institute of Physics, Chinese Academy of Sciences, Beijing 100080, China
}

(Dated: February 9, 2020)

\begin{abstract}
We investigate localized atomic matter waves in two-component Bose-Einstein condensates coupled by the two photon microwave field. Interestingly, the oscillations of localized atomic matter waves will gradually decay and finally become non-oscillating behavior even if existing coupling field. In particular, atom numbers occupied in two different hyperfine spin states will appear asymmetric occupations after some time evolution.
\end{abstract}

PACS numbers: 03.75.-b,67.40.-w,39.25.+k

The creation of dark solitons [1, 2, 3] and bright solitons [4, 5] in trapped weakly interacting atomic gases has opened the road to understanding and controlling nonlinear properties of atomic matter waves. Although many nonlinear phenomena in Bose-Einstein condensates (BECs) may have their counterparts in nonlinear optics, unique manageability of the properties of BECs, new setups can be studied, which were not realized in nonlinear optics. This opens the promising perspective for numerous applications of the nonlinear matter-wave physics such as atom chip and quantum information processing on the nanometer scale. The s-wave scattering interaction in the BECs is the determining factor (attractive for bright solitons, repulsive for dark solitons) and attractive interaction leads to condensates collapse in effective two- and three- dimensional cases if the atomic number exceeds a critical value, and to soliton formation in quasi one-dimensional (1D) traps. In the quasi-1D regime, two condensates in different hyperfine states reduce to coupled Gross-Pitaevskii equations (GPEs) [6, 7]. In the system of optically coupled two-component BECs, some beautiful work [8, 9, 10] has discussed Josephson-type density evolution process and pointed out the internal tunnelling effects of the two optically coupled condensates.

In the present Letter, we consider two-component Bose-Einstein condensates coupled by two photon microwave field. Using the soliton as staring spatial pattern formation, we study dynamic behavior of two components condensates under tuning atomic interactions. Surprisingly, the spatial shape of condensates presents very localized all the time even if changing the atomic interactions. During the transmission process, two localized atomic matter waves will collide each other many times and exchange energy greatly. In particular, oscillations of atom numbers of two components are very unstable and will periodically decay to zero, and the speed of decay can also be controlled through tuning atomic interactions. Moreover, atom numbers occupied in two different hyperfine spin states will appear asymmetric occupations in the end.
Experimentally, the bright soliton have been successfully produced from ${ }^{7} \mathrm{Li}$ atoms in the internal atomic hyperfine spin state $\left|F=1, m_{f}=1\right\rangle[\underline{4},[5]$. Theoretically, we consider two different hyperfine spin state with attractive interactions denoted as $\Psi_{1}$ and $\Psi_{2}$, respectively. The ratio of the radial and the axial trapping frequency of harmonic potential obeys $\omega_{r} / \omega_{z} \gg 1$, for example, $\left.\omega_{r} / \omega_{z}=5.1 \times 10^{3}\right)$ [4], so two hyperfine spin states are both in the transverse ground state. The condensate wave function is normalized as $\int d r\left(\left|\Psi_{1}\right|^{2}+\left|\Psi_{2}\right|^{2}\right)=$ $N_{1}+N_{2}=N$, where $N$ is the total number of atom in two components. The attractive intra- and interatomic interactions are $U_{i i}=-4 \pi \hbar^{2}\left|a_{i i}\right|\left|\Psi_{i}\right|^{2} / m$ and $U_{i j}=-4 \pi \hbar^{2}\left|a_{i j}\right|\left|\Psi_{i}\right|^{2} / m(i, j=1,2)$, where $-\left|a_{i i}\right|$ and $-\left|a_{i j}\right|$ is the intra-species and inter-species s-wave scattering lengths, $\mathrm{m}$ is the atomic mass of the ${ }^{7} \mathrm{Li}$ atom. In one-dimensional case the effective couplings $\bar{U}_{i i}$ and $\bar{U}_{i j}$ is represented by $\bar{U}_{i i}=-2 \hbar^{2}\left|a_{i i}\right|\left|\Psi_{i}\right|^{2} / m a_{r}^{2}$ and $\bar{U}_{i j}$ $=-2 \hbar^{2}\left|a_{i j}\right|\left|\Psi_{i}\right|^{2} / m a_{r}^{2}$ where $a_{r}=\sqrt{\hbar / m \omega_{r}}$ [1]. After tuning the strength of interaction to a sufficiently large negative value through use of atomic Feshbach resonance [12, 13], the condensates can be set free along the waveguide, i.e. $\omega_{z}=0$. The atomic transition between two-component BECs is induced by the two photon microwave field with the effective Rabi frequency $\Omega$ and a finite detune $\delta$, where the $\Omega$ and $\delta$ are independent of both time and axial coordinate as experimental case [14].

In view of the difference of inter- and intra-atomic interactions, it is convenient to describe the two-component BECs coupled with the two photon microwave field in the following dimensionless coupled GPEs,

$$
\begin{aligned}
& i \frac{\partial \phi_{i}}{\partial t}=-\frac{\partial^{2} \phi_{i}}{\partial z^{2}}-\frac{4 N\left|a_{i j}\right|}{a_{r}}\left(\left|\phi_{i}\right|^{2}+\left|\phi_{j}\right|^{2}\right) \phi_{i}-\frac{\delta}{\omega_{r}} \cos (\pi i) \phi_{i} \\
& +\frac{\Omega}{\omega_{r}} \phi_{j}-C_{i} \frac{4 N\left|a_{i j}\right|}{a_{r}}\left|\phi_{i}\right|^{2} \phi_{i} .
\end{aligned}
$$

where $i, j=1,2, i \neq j$ and time $\mathrm{t}$ and coordinate $\mathrm{z}$ are measured in units $2 / \omega_{r}$ and $a_{r}=\sqrt{\hbar / m \omega_{r}}$, respectively, so, energy is in unit of $\hbar \omega_{r} / 2, C_{i}=\left(\left|a_{i i}\right|-\right.$ $\left.\left|a_{i j}\right|\right) /|a i j|$ measure the difference of interaction strength, and $\int d z\left(\left|\phi_{1}\right|^{2}+\left|\phi_{2}\right|^{2}\right)=1$ is normalization condition. 
In order to compare with experimental case, some parameters in equations (11) are chosen as $\omega_{r}=2 \pi \times 625$ $H z\left(\mathrm{so}, a_{r} \approx 1.51 \mu \mathrm{m}\right),\left|a_{i j}\right|=0.16 \mathrm{~nm}$ (same as [4]). Since $a_{r} /\left|a_{i j}\right| \approx 9437$ [15, 16, 17, 18], so we choose the number of ${ }^{7} \mathrm{Li}$ atom $N=6000$, and so coefficient $4 N\left|a_{i j}\right| / a_{r} \approx 2.5$.

In the special case of $C_{i}=0$, i.e. $\left|a_{i i}\right|=\left|a_{i j}\right|$, equations (11) have exactly two-soliton solutions

$$
\begin{aligned}
\phi_{i}= & {\left[\operatorname { s i n } ( \frac { \pi i } { 2 } - \theta ) e ^ { i \Gamma t } \left(\alpha_{i} e^{\eta_{i}}+\alpha_{j} e^{\eta_{j}}+e^{\delta_{i}+\eta_{i}+\eta_{i}^{*}+\eta_{j}}\right.\right.} \\
& \left.+e^{\delta_{j}+\eta_{i}+\eta_{j}+\eta_{j}^{*}}\right)-\cos \left(\frac{\pi i}{2}-\theta\right) e^{-i \Gamma t}\left(\beta_{i} e^{\eta_{i}}+\beta_{j} e^{\eta_{j}}\right. \\
& \left.\left.+e^{\delta_{i}+\eta_{i}+\eta_{i}^{*}+\eta_{j}}+e^{\delta_{j}+\eta_{i}+\eta_{j}+\eta_{j}^{*}}\right)\right] / D .
\end{aligned}
$$

here $i, j=1,2, i \neq j$ and $\theta=\tan ^{-1}\left(\frac{\Omega}{\delta}\right) / 2, \Gamma=\left[\left(\delta / \omega_{r}\right)^{2}+\right.$ $\left.\left(\Omega / \omega_{r}\right)^{2}\right]^{1 / 2}, \eta_{j}=k_{j}\left(z+i k_{j} t\right), D=1+e^{\eta_{1}+\eta_{1}^{*}+R_{1}}+$ $e^{\eta_{1}+\eta_{2}^{*}+\delta_{0}}+e^{\eta_{1}^{*}+\eta_{2}+\delta_{0}^{*}}+e^{\eta_{2}+\eta_{2}^{*}+R_{2}}+e^{\eta_{1}+\eta_{1}^{*}+\eta_{2}+\eta_{2}^{*}+R_{3}}$, $e^{\delta_{0}}=\kappa_{12} /\left(k_{1}+k_{2}^{*}\right), e^{R_{j}}=\kappa_{j j} /\left(k_{j}+k_{j}^{*}\right), e^{\delta_{i}}=$ $\left(k_{i}-k_{j}\right)\left(\alpha_{i} \kappa_{j i}-\alpha_{j} \kappa_{i i}\right) /\left(k_{i}+k_{i}^{*}\right)\left(k_{i}^{*}+k_{j}\right), e^{\delta_{i}^{\prime}}=$ $\left(k_{i}-k_{j}\right)\left(\beta_{i} \kappa_{j i}-\beta_{j} \kappa_{i i}\right) /\left(k_{i}+k_{i}^{*}\right)\left(k_{i}^{*}+k_{j}\right), e^{R_{3}}=$ $\left|k_{1}-k_{2}\right|^{2}\left(\kappa_{11} \kappa_{22}-\kappa_{12} \kappa_{21}\right) /\left(k_{1}+k_{1}^{*}\right)\left(k_{2}+k_{2}^{*}\right) \mid k_{1}+$ $\left.k_{2}^{*}\right|^{2}$, and $\kappa_{i j}=\left[\left(2 N\left|a_{12}\right| / a_{r}\right)\left(\alpha_{i} \alpha_{j}^{*}+\beta_{i} \beta_{j}^{*}\right)\right]\left[\left(k_{i}+\right.\right.$ $\left.\left.k_{j}^{*}\right)\right] .\left[\left(\alpha_{1}, \beta_{1}\right)\left\{\kappa_{11}^{-1}, \kappa_{12}^{-1}\right\}-\left(\alpha_{2}, \beta_{2}\right)\left\{\kappa_{21}^{-1}, \kappa_{22}^{-1}\right\}\right]$

In order to facilitate the understanding of the influences of microwave field on solitons, it is convenient to obtain the asymptotic forms of solutions (2) of Eq. (1)

$S_{j}^{n \pm}=\left[\sin \left(\frac{\pi j}{2}-\theta\right) e^{i \Gamma t} A_{j}^{n \pm}-\cos \left(\frac{\pi j}{2}-\theta\right) e^{-i \Gamma t} A_{j}^{n \pm}\right] \phi^{n \pm}$.

here superscripts in $S_{j}^{n \pm}(j, n=1,2)$ denote solitons in limit $t \rightarrow \pm \infty$, subscripts refer to the components and $\left(\phi^{1-}, \phi^{2+}\right)=\left\{k_{1 R} e^{i \eta_{1 I}} \operatorname{sech}\left(\eta_{1 R}+\right.\right.$ $\left.\left.R_{1} / 2\right), \quad k_{2 R} e^{i \eta_{2 I}} \operatorname{sech}\left(\eta_{2 R}+R_{2} / 2\right)\right\}, \quad\left(\phi^{2-}, \phi^{1+}\right)$

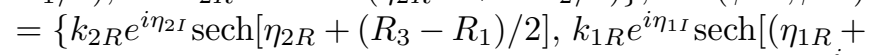
$\left.\left.\left(R_{3}-R_{1}\right) / 2\right]\right\}$, and the polarization vectors $A_{i}^{j-}$ and $A_{i}^{j+}$ are defined as $\left\{\left(A_{1}^{1-}, A_{2}^{1-}\right),\left(A_{1}^{2+}, A_{2}^{2+}\right)\right\}=$ $\left[\left(2 N\left|a_{12}\right| / a_{r}\right)\left(\left|\alpha_{1}\right|^{2}+\left|\beta_{1}\right|^{2}\right)\right]^{\frac{1}{2}}\left\{\left(\alpha_{1}, \beta_{1}\right),\left(\alpha_{2}, \beta_{2}\right)\right\}$ and $\left\{\left(A_{1}^{2-}, A_{2}^{2-}\right),\left(A_{1}^{1+}, A_{2}^{1+}\right)\right\}=c\left[\left(2 N\left|a_{12}\right| / a_{r}\right)\left(\left|\alpha_{1}\right|^{2}+\right.\right.$ $\left.\left.\left|\beta_{1}\right|^{2}\right)^{\frac{1}{2}}\right]\left\{\left(a_{1} / a_{1}^{*}\right),\left(a_{2} / a_{2}^{*}\right)\right\}\left\{\left(\alpha_{1} \kappa_{11}^{-1}-\alpha_{2} \kappa_{21}^{-1}, \beta_{1} \kappa_{11}^{-1}-\right.\right.$ $\left.\left.\beta_{2} \kappa_{21}^{-1}\right),\left(\alpha_{1} \kappa_{12}^{-1}-\alpha_{2} \kappa_{22}^{-1}, \beta_{1} \kappa_{12}^{-1}-\beta_{2} \kappa_{22}^{-1}\right)\right\} \quad$ in which $c=\left(1 /\left|\kappa_{12}\right|^{2}-1 / \kappa_{11} \kappa_{22}\right)^{-\frac{1}{2}}, \quad a_{i}=$ $\left(k_{i}+k_{j}^{*}\right)\left[\cos (\pi i+\pi)\left(k_{i}-k_{j}\right)\left(\alpha_{i}^{*} \alpha_{j}+\beta_{i}^{*} \beta_{j}\right)\right]^{\frac{1}{2}}$.

It is easy to see from the Eqs. (3) that the density of solitons are $\left|S_{l}^{n \mp}(z, t)\right|^{2}=\left[\left|A_{l}^{n \mp}\right|^{2} \cos ^{2} \theta+\left|A_{j}^{n \mp}\right|^{2} \sin ^{2} \theta+\right.$ $\left.(-1)^{l}\left|A_{l}^{n \mp}\right|\left|A_{j}^{n \mp}\right| \sin 2 \theta \cos \left(2 \Gamma t+\varphi^{n \mp}\right)\right]\left|\phi^{n \mp}\right|^{2}, n, l, j=$ $1,2, \quad l \neq j$, where $\varphi^{n \mp}=\tan ^{-1}\left(A_{1 I}^{n \mp} / A_{1 R}^{n \mp}\right)-$ $\tan ^{-1}\left(A_{2 I}^{n \mp} / A_{2 R}^{n \mp}\right)$. So, in this special case, the reason of density oscillation is just because of the presence of time dependence part $(-1)^{l}\left|A_{l}^{n \mp}\right|\left|A_{j}^{n \mp}\right| \sin 2 \theta \cos (2 \Gamma t+$ $\left.\varphi^{n \mp}\right) \phi^{n \mp}(z, t)^{2}$, where the frequency of oscillation is $2 \Gamma$ and can be tuned easily through changing the effective Rabi frequency $\Omega$, a finite detune $\delta$ and radial trapping frequency $\omega_{r}$. In particular, when $\theta=n \pi / 2(n \in$ even $)$ or $\Gamma=0$, the oscillation will disappear.

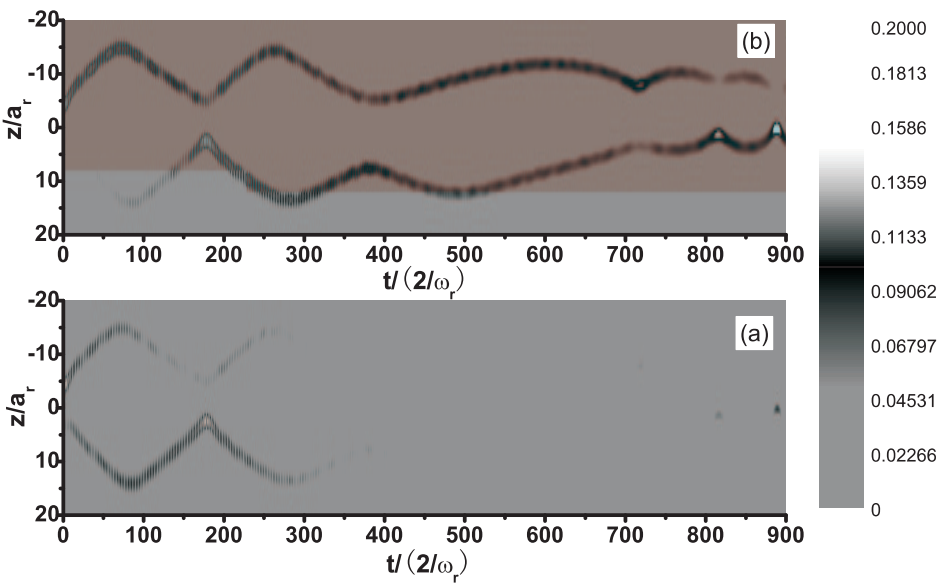

FIG. 1: Time development of the condensate densities $(a)$ $\left|\phi_{1}\right|^{2}$, (b) $\left|\phi_{2}\right|^{2}$ along the $z$ axis for $N=6000, \omega_{r}=2 \pi \times$ $625 \mathrm{~Hz}, a_{r} \approx 1.51 \mu \mathrm{m}, \quad \delta=0.25 \omega_{r}$ and $\Omega=0.75 \omega_{r}$, $a_{11}=-0.24 \mathrm{~nm}, a_{12}=-0.16 \mathrm{~nm}, a_{22}=-0.32 \mathrm{~nm}$. The chosen parameters for initial solitons are $k_{1}=0.313+0.2 i$, $k_{2}=0.313-0.2 i, \alpha_{1}=\beta_{1}=\beta_{2}=0.251+0.966 i, \alpha_{2}=$ $0.966+0.251 i$.

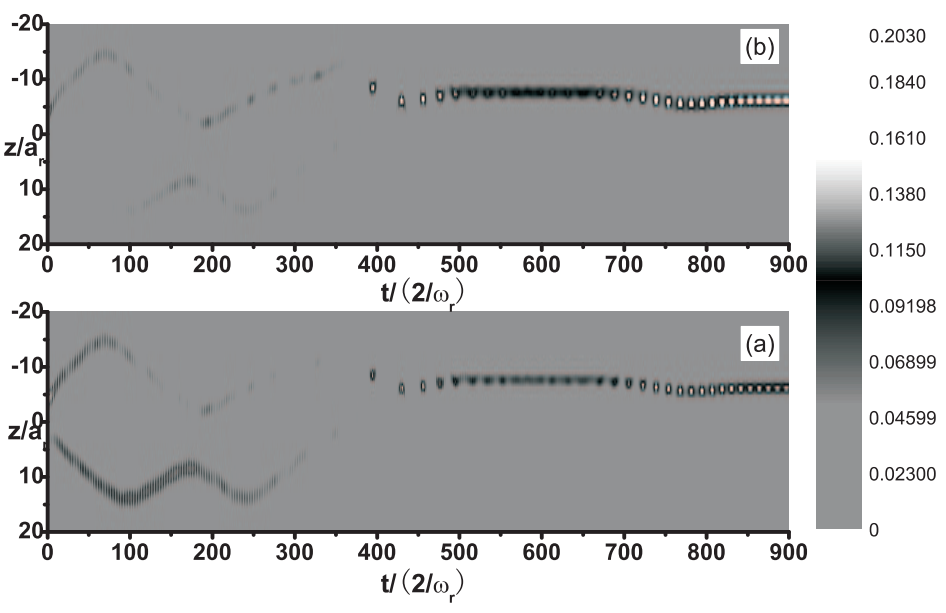

FIG. 2: Time development of the condensate densities $(a)$ $\left|\phi_{1}\right|^{2}$, (b) $\left|\phi_{2}\right|^{2}$ along the $z$ axis for $N=6000, \omega_{r}=2 \pi \times$ $625 \mathrm{~Hz}, a_{r} \approx 1.51 \mu \mathrm{m}, \quad \delta=0.25 \omega_{r}$ and $\Omega=0.75 \omega_{r}$, $a_{11}=-0.32 \mathrm{~nm}, a_{12}=-0.16 \mathrm{~nm}, a_{22}=-0.24 \mathrm{~nm}$. The chosen parameters for initial solitons are $k_{1}=0.313+0.2 i$, $k_{2}=0.313-0.2 i, \alpha_{1}=\beta_{1}=\beta_{2}=0.251+0.966 i, \alpha_{2}=$ $0.966+0.251 i$.

More interesting case is see the dynamics caused by changing the nonlinearities, i.e. atomic interactions. Experimentally, changing the nonlinearity can be realized by Feshbach resonance. In this system, changing the nonlinearity corresponds to change $C_{i}$ in equations (11). So, we will investigate pattern formation under changing $C_{i}$. We perform numerical simulations of Eq. (11) based on discretizing it in both space and time using the known boundary conditions, e.g., $\left|\phi_{j}\left(z_{\min }, t\right)\right|=$ $\left|\phi_{j}\left(z_{\max }, t\right)\right|=0$ where $z_{\min }=-20$ and $z_{\max }=20$. 
Initial states we prepared are two soliton solutions (2) of Eq. (11). The mismatch of $a_{i i}$ with $a_{i j}$ in initial configuration will result in perturbations of the condensates. In our numerical simulations, the parameters are chosen as $k_{1}=0.313+0.2 i, k_{2}=0.313-0.2 i$, $\alpha_{1}=\beta_{1}=\beta_{2}=0.251+0.966 i, \alpha_{2}=0.966+0.251 i$, $\delta=0.25 \Omega_{r}$ and $\Omega=0.75 \omega_{r}$. So, the frequency of oscillations is $2 \Gamma=2\left[\left(\delta / \omega_{r}\right)^{2}+\left(\Omega / \omega_{r}\right)^{2}\right]^{1 / 2}=1.58$ corresponding to $0.8 \mathrm{msec}$ considering time units.

Figure 1 shows image of initial conditions (2) propagating about 560 periods for $\left|a_{11}\right|=1.5\left|a_{12}\right|=0.24$ $\mathrm{nm}$ and $\left|a_{22}\right|=2\left|a_{12}\right|=0.32 \mathrm{~nm}$. It is interesting to point out that in our numerical simulations, spatial pattern formation all the time preserves soliton-like shaped i.e., localized atomic matter waves during transmission process. Although introduced important the mismatch of $a_{i i}$ with $a_{i j}$, their influences on dynamics of initial solitons are only velocity and amplitude. That is to say, increasing the absolute value of atomic interactions will make the condensates more local instead of destroying localized density distributions.

As is shown in Figure1, in the beginning, the densities of two localized atomic matter waves are oscillate periodically and later, oscillations will quickly damp out for each components as shown in Fig. 1(a) and Fig. 1(b), respectively. The total density of two components is well conserved during the transmission process, but the densities of each components will change significantly. This is very incompatible with special case $\left|a_{11}\right|=\left|a_{12}\right|=\left|a_{22}\right|$.

One of the most different phenomenon from special case is that two localized waves for each component no longer transmit separately and on the contrary, they transmit closer and closer. We can see that the two localized waves collide for the first time at about $90 \mathrm{msec}$. After the collision, the relative density of one of waves is enhanced and the other is suppressed obviously. As shown in Figure 1, the density of component one corresponding to Fig. 1(a) is suppressed and the other corresponding to Fig. 1(b) is enhanced at the same time. So, the interaction between two waves can be used to transfer energy from one to the other. At about $190 \mathrm{msec}$, the two waves collide for the second time and oscillations are very weak now. Subsequently, the two waves will keep to collide faster and become closer and closer.

It must be pointed out that whether the atomic interactions have the same values or not, the two localized atomic matter waves for component one are always completely overlapped with the others for component two in configuration space. So, from the experimental point of view, observable phenomena are always the combination effects of two components. The oscillations effect as shown in Fig. 1(a) and Fig. 1(b) can be observed through kicking BECs so that different hyperfine spin components will be separated in configuration space.

In two different hyperfine spin components, two localized atomic matter waves are move closer and closer after
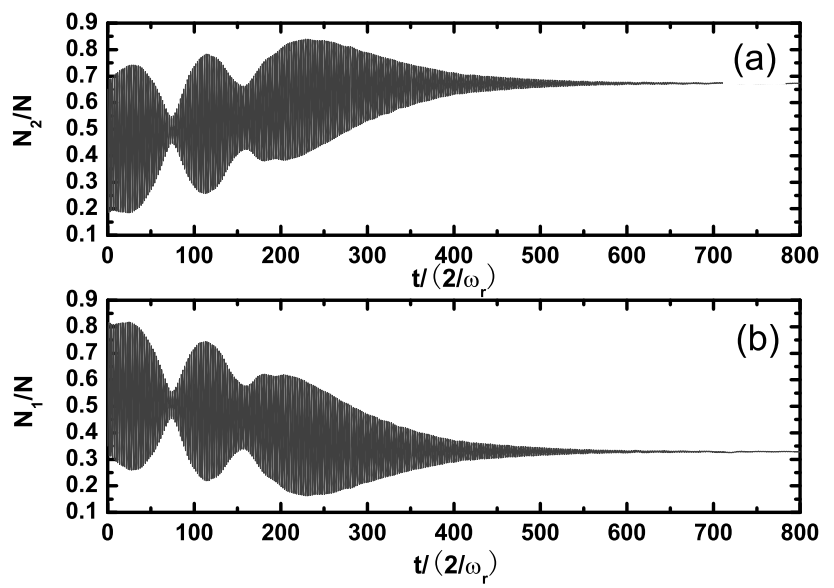

FIG. 3: Time development of the atom numbers (a) component $\phi_{1},(b)$ component $\phi_{2}$ for $N=6000, \omega_{r}=2 \pi \times 625 \mathrm{~Hz}$, $a_{r} \approx 1.51 \mu \mathrm{m}, \quad \delta=0.25 \omega_{r}$ and $\Omega=0.75 \omega_{r}, a_{11}=-0.24$ $\mathrm{nm}, a_{12}=-0.16 \mathrm{~nm}, a_{22}=-0.32 \mathrm{~nm}$. The chosen parameters for initial solitons are $k_{1}=0.313+0.2 i, k_{2}=0.313-0.2 i$, $\alpha_{1}=\beta_{1}=\beta_{2}=0.251+0.966 i, \alpha_{2}=0.966+0.251 i$.

a few collisions. So, now the questions are can we make two waves close faster and transfer energy more efficient? In Figure 1, the atomic s-wave scattering lengths are chosen as $a_{11}=-0.24 \mathrm{~nm}, a_{12}=-0.16 \mathrm{~nm}, a_{22}=-0.32$ $n m$ and the relation of absolute value of $a_{11}$ and $a_{22}$ is $\left|a_{11}\right|<\left|a_{22}\right|$. So, it is naturally to investigate the time development of condensates in condition of inversing the relation of $\left|a_{11}\right|$ and $\left|a_{22}\right|$, i.e. $\left|a_{11}\right|>\left|a_{22}\right|$. It is very surprising that two localized waves not only close faster but also transfer energy more efficient than former case. This is illustrated in Figure 2. The atomic s-wave scattering lengths are chosen as $a_{11}=-0.32 \mathrm{~nm}, a_{12}=-0.16 \mathrm{~nm}$, $a_{22}=-0.24 \mathrm{~nm}$, and the other parameters are chose as the same as before.

Fig. 2(a) and Fig. 2(b) show the time development of the condensate densities $\left|\phi_{1}\right|^{2}$ and $\left|\phi_{2}\right|^{2}$ along the $z$ axis, respectively. Due to changing the relation from $\left|a_{11}\right|<\left|a_{22}\right|$ to $\left|a_{11}\right|>\left|a_{22}\right|$, the density of one of localized waves has enhanced greatly and the other has suppressed extremely only after the second time collision. And the density of enhanced localized wave of each component will no longer increase or decrease during transmission process after the second time collision. Moveover, enhanced localized wave of each component will fixed in the position all the time and the weaken localized wave will move towards the boundary after the second time collision. The future applications of these effects can be existed in atom optics, atom transport and quantum information.

Another interesting problem is the effects of chang- 


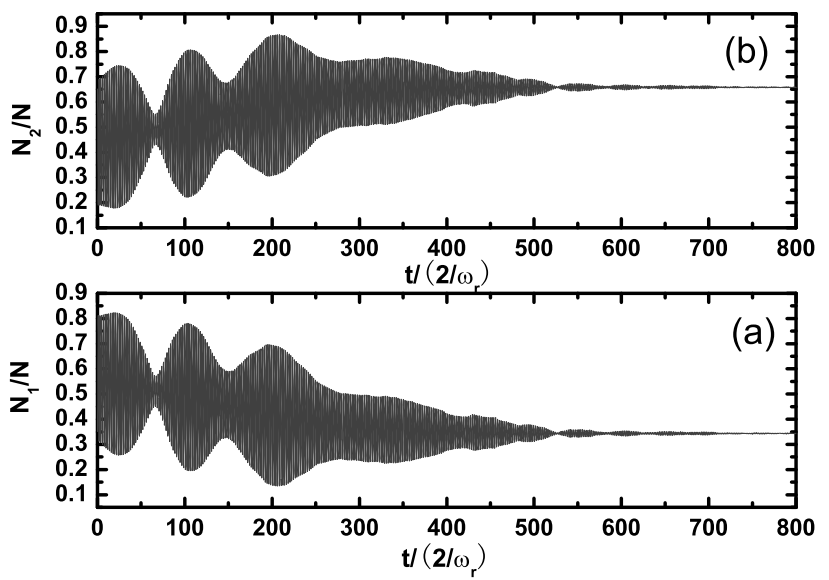

FIG. 4: Time development of the atom numbers (a) component $\phi_{1},(b)$ component $\phi_{2}$ for $N=6000, \omega_{r}=2 \pi \times 625 \mathrm{~Hz}$, $a_{r} \approx 1.51 \mu \mathrm{m}, \quad \delta=0.25 \omega_{r}$ and $\Omega=0.75 \omega_{r}, a_{11}=-0.32$ $\mathrm{nm}, a_{12}=-0.16 \mathrm{~nm}, a_{22}=-0.24 \mathrm{~nm}$. The chosen parameters for initial solitons are $k_{1}=0.313+0.2 i, k_{2}=0.313-0.2 i$, $\alpha_{1}=\beta_{1}=\beta_{2}=0.251+0.966 i, \alpha_{2}=0.966+0.251 i$.

ing atomic interactions on atom numbers of each components. From Eq. (3), it is easier to see that due to applied coupling field, atom numbers of each hyperfine component will exchange periodically. Naturally, the question is whether the persistent oscillations can still be observed or not when tuning intra- and inter- atomic interactions. Figure 3 and 4 show the time development of atom numbers of each component for different atomic interactions. In Figure 3, as the system evolves, oscillations of each component both enhanced gradually in the beginning. At about $10 \mathrm{msec}$, the intensities of oscillations of each component both achieve maximum, and then begin to decrease, until at about $38 \mathrm{msec}$ achieve minimum. At time interval of $38 \mathrm{msec}$ to $80 \mathrm{msec}$, the intensities of oscillations again change from maximum to minimum. Then, it is very surprising that after achieving maximum for the third time at about $115 \mathrm{msec}$, oscillations will gradually damp out and disappear finally. Moreover, it is interesting to notice that more atom number occupies the hyperfine spin state $\phi_{2}$ as shown in Fig. 3(b) and less atom number occupies the other hyperfine spin state $\phi_{1}$ as shown in Fig. 3(a) when oscillations disappear. The ratio of atom numbers of each component is $0.32: 0.68$.

The same effects can be observed in Figure 4 . When the relation of atomic interactions changes as shown in Figure 4, oscillations will experience more periods than former case before damp out. The ratio of atom numbers of each component is $0.35: 0.65$ and slightly different from former case.

So, the conclusion is that atomic interactions have strong effects on exchange of atom numbers in this system. Through tuning atomic interactions using Feshbach resonance, we can control relative atom numbers occupied different hyperfine spin states and their oscillations. That is to say, we can control total magnetic moment of condensates using above mentioned effects. It is very fascinating to observe these effects experimentally.

In this Letter, we have found a new type of wave localized atomic matter waves in two-component BECs coupled with the two photon microwave field. By tuning the atomic interactions, localized atomic matter waves will experience a damped oscillations. During the transmission process, two localized atomic matter waves will collide many times and exchange energy significantly. In particular, the atom numbers occupied different hyperfine spin states will appear asymmetric transition finally. Our result gives some fascinating effects on dynamics which are unobserved in real experiments so far. This work may be important for controllable nonlinear atom optics.

This work was supported by the NSF of China under grant 90403034, 90406017, 60525417; and the National Key Basic Research Special Foundation of China under 2005CB724508 and 2006CB921400.

[1] W. P. Reinhardt et al., J. Phys. B 30, L785 (1997).

[2] S. A. Morgan et al., Phys. Rev. A 55, 4338 (1997).

[3] T. Busch et al., Phys. Rev. Lett. 87, 010401 (2001); Z. X. Liang, Z. D. Zhang, W. M. Liu, Phys. Rev. Lett. 94, 050402 (2005).

[4] K. E. Strecker et al., Nature 417, 150 (2002).

[5] L. Khaykovich et al., Science 296, 1290 (2002).

[6] T. Busch et al., Phys. Rev. Lett. 84, 2298 (2000); W. M. Liu, B. Wu, Q. Niu, Phys. Rev. Lett. 84, 2294 (2000).

[7] M. Modugno et al., Phys. Rev. E 62, 063607 (2000); W. M. Liu, W. B. Fan, W. M. Zheng, J. Q. Liang, S. T. Chui, Phys. Rev. Lett. 88, 170408 (2002).

[8] J. Williams et al., Phys. Rev. A 59, R31 (1999).

[9] Q. H. Park et al., Phys. Rev. Lett. 85, 4195 (2000).

[10] S. Kohler et al., Phys. Rev. Lett. 89, 060403 (2002).

[11] M. Olshanii, Phys. Rev. Lett. 81, 938 (1998).

[12] S. Inouye et al., Science 393, 151 (1998).

[13] S. L. Cornish et al., Phys. Rev. Lett. 85, 1795 (2000).

[14] M. R. Matthews et al., Phys. Rev. Lett. 81, 243 (1998).

[15] V. Pérez-Garćia et al., Phys. Rev. A 57, 3837 (1998).

[16] A. E. Muryshev et al., Phys. Rev. A 60, R2665 (1999).

[17] L. D. Carr et al., J. Phys. B 33, 3983 (2000).

[18] Y. S. Kivshar et al., Phys. Lett. A 278, 225 (2001). 